\title{
A facile access to condensed and spirosubstituted pyrimidine phosphor esters
}

\author{
Wafaa M. Abdou, ${ }^{a^{*}}$ Mounir A. I. Salem, ${ }^{\text {and Reham F. Barghash }}{ }^{\mathrm{a}}$ \\ ${ }^{a}$ Chemical Industries Division, National Research Centre, Dokki, D -12622, Cairo, Egypt \\ ${ }^{b}$ Department of Chemistry, Faculty of Science, Ain-Shams University, Cairo, Egypt \\ E-mail: wabdou@intouch.com
}

\section{Dedicated to the memory of Professor Charles Rees}

\begin{abstract}
Treatment of alloxan-5-oximes with different types of phosphonate carbanions gave moderate to high yields of condensed and spiro bis-heterocyclic systems bearing a phosphonate substituent. Mechanisms for the formation of the five- and six-membered rings were provided. A marked resemblance between alloxan-5-oxime and 1,3-dimethyl derivative is observed through the studied reactions. The bioassay indicated that some of the products have good selective antitumor activity and the structure-activity relationship (SAR) of new phosphonates has also been studied.
\end{abstract}

Keywords: Alloxan-5-oximes, substituted bis-heterocycle phosphor esters, Wittig-Horner carbanions, pyrimidines, anti-tumor activity, structure-activity relationship (SAR)

\section{Introduction}

Recently, a novel heterocyclic transformation reaction has been described involving an additionelimination, and rearrangement sequence of violuric acid (1) with saturated, unsaturated and active alkylidenephosphoranes leading to a series of oxazolo-pyrimidenes as well as pyrimidooxazines (Scheme 1). ${ }^{1}$ It has been pointed out that the reactions of such ylides proceeded only when the latter were generated in situ from the corresponding salts (see Scheme 1) in the presence of $\sim 3$-fold excess $\mathrm{LiOH}$ (aqueous). The findings reflected the inertness of molecule $\mathbf{1}$, which was attributed to the low reactivity of the oxime function and the amidic carbonyl group toward nucleophilic attack. The thermal condition, coupled with the presence of an excess of a strong base that used for the generation of ylide, however, can deprotonate either the ylides or the oxime 1 promoting thus the further reaction. Furthermore, the adjacent carbonyl group re- 
enforces the electrophilic character of the oximino moiety. Preliminary screening of the products showed appreciable potency in antagonizing hypothermia and catalepsy caused by reserpine. ${ }^{1}$

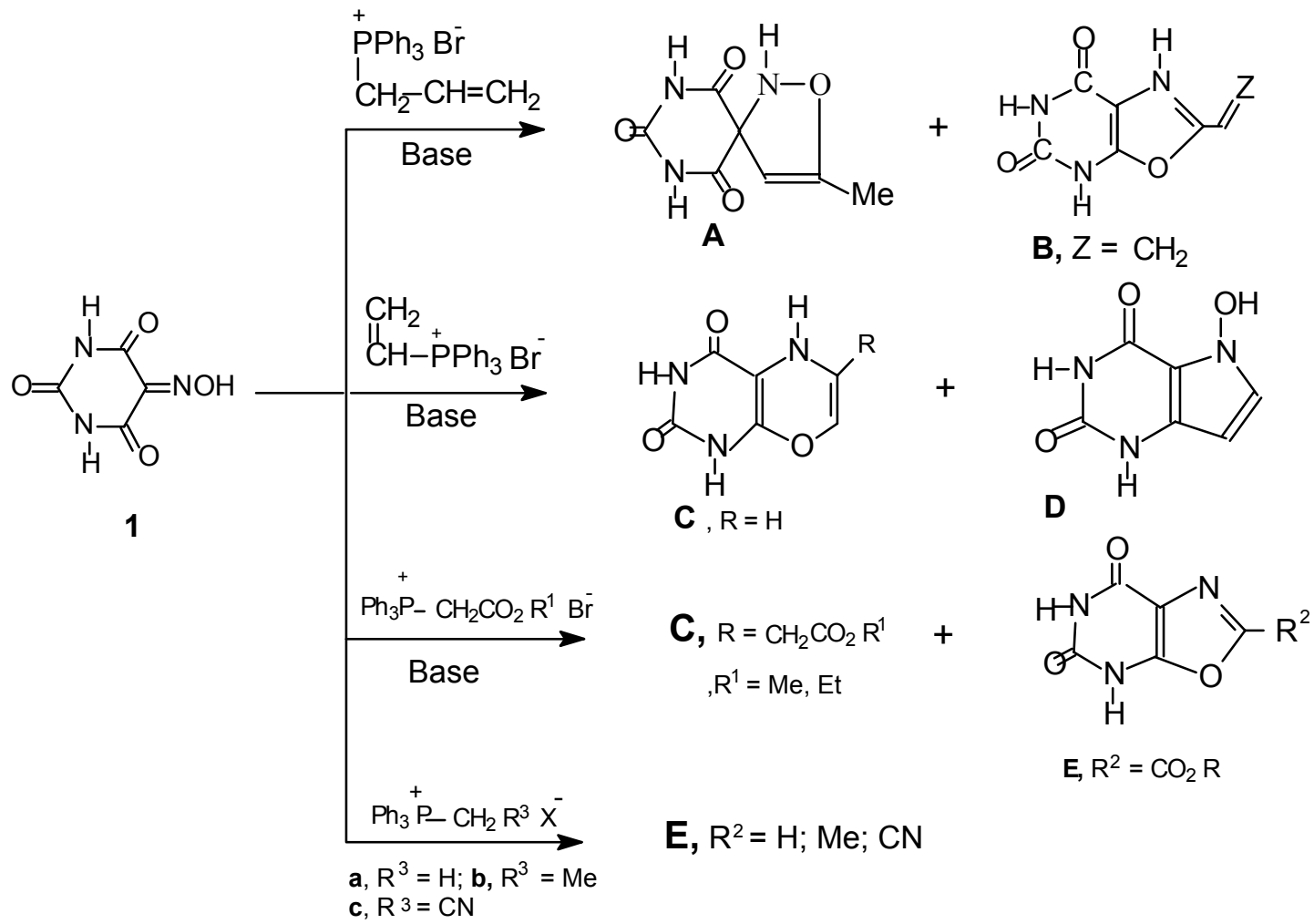

\section{Scheme 1}

In contribution of this work, the present study has been focused on synthesis of bioactive heterocyclic systems bearing phosphonate substituent. The methodology centered on the application of phosphonyl carbanions (Wittig-Horner (WH) reagents) to violuric acid (1) and the dimethyl derivative 2. Pharmacological evaluation of the produced phosphonates is discussed. Furthermore, a comparative study on the behavior of $\mathbf{1}$ toward alkylidenephosphoranes counterparts is also undertaken.

2,4,6-Trioxo-5-oximinohexylhydropyrimidine (1, also known as violuric acid, barbituric acid-5-oxime, and alloxan-5-oxime) is an example of 6-membered ambident heterocycle possessing several tautomeric structures (Figure 1) and display dual reactivity., ${ }^{2,3}$ Violuric acid is almost colorless, or at best only a pale yellow color, in the solid state due to its existence in the oximino-ketonic formula 1a. ${ }^{3}$ In an aqueous solution it exists mainly in form 1a with only a small proportion of the nitroso-enolic structure $\mathbf{1 b}$. Nevertheless, as soon as an alkali like $\mathrm{NaOH}$ is added to the solution, the color turns to deep brown indicating that the original weekly acidic oximino-ketonic character of violuric acid tautomerizes to the more acidic nitroso-enolic form 1b. And in this form it becomes fixed by salt formation with sodium hydroxide. ${ }^{3}$ 


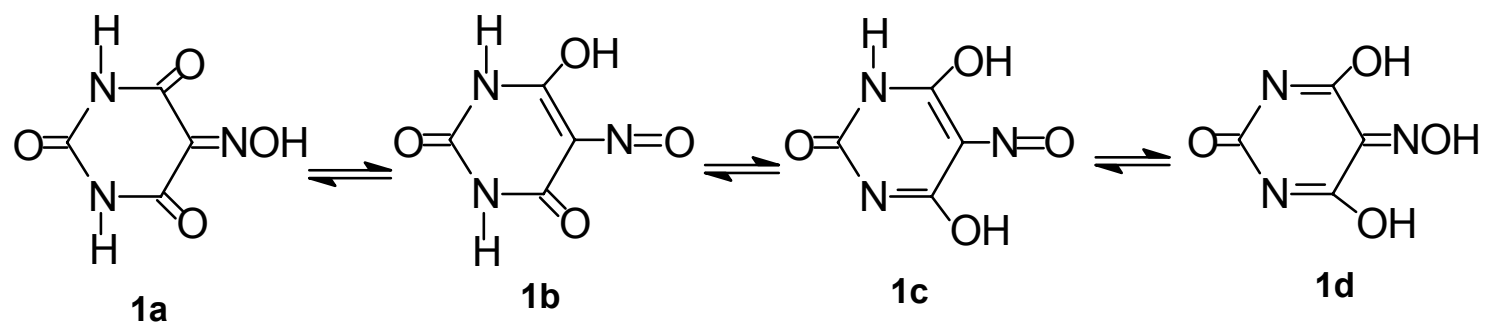

\section{Figure 1}

\section{Results and Discussion}

The procedure of the reactions of oximes $\mathbf{1}$ and $\mathbf{2}$ with WH reagents are analogous to that of the latter with carbonyl compounds. ${ }^{4}$ Typically, an ethanol solution of 1.5 equivalent of the phosphorus reagent was treated with a solution of sodium ethanolate (EtONa, 4.5 equiv.) to generate the carbanion, and finally the oxime substrate was added. The reaction mixture was refluxed for the proper time (TLC), poured into distilled water and acidified with $\mathrm{HCl}(1 \mathrm{~N})$. The product mixture was easily separated by solvent extraction, and purified by chromatography. The formed adducts are depicted in Schemes 2-6.

According to this procedure, alloxan-5-oxime (1) reacted with 1.5 molar amount of methyl diethyl phosphonoacetate (3a) in ethanol solution containing EtONa yielding two crystalline compounds. The same materials were also isolated when 1 was allowed to react with triethyl phosphonoacetate (3b) under similar conditions. These were formulated as diethyl $(2,5,6,7,8$ pentahydro-2,5,7-trioxopyrimidino[4,5-b][1,4] oxazin-3yl)phosphonate $(\mathbf{5 a}, 35 \%$ yield) and diethyl (2`-ethyl-5 '-hydroxy 1,2,3,4,6-pentahydro-2,4,6-trioxospiro[pyrimidine [5,3 $][1,2]$ oxazole]-4 yl)phosphonate (8a, 28\% yield). Similar treatment of 1,3-dimethyl barbituric acid-5-oxime (2) with $\mathbf{3 a}$ or $\mathbf{3 b}$ afforded, in both cases the parallel analogs diethyl (6,8-dimethyl-2,5,7-trihydro-2,5,7-trioxopyrimidino[4,5-b][1,4]oxazin-3yl)-phosphonate $\quad \mathbf{5 b}$ (38\%) and diethyl (1,3-dimethyl-2'-ethyl-5'-hydroxy -2,4,6-trihydro-2,4,6-trioxospiro[pyrimidine[5,3`][1,2] oxazole]-4'yl)phosphonate (8b) (29\%) (Scheme 2). Structures 5 and 8 were substantiated on the basis of their elemental analyses, IR, ${ }^{31} \mathrm{P}-,{ }^{1} \mathrm{H}-,{ }^{13} \mathrm{C}$ NMR and mass spectral data. 


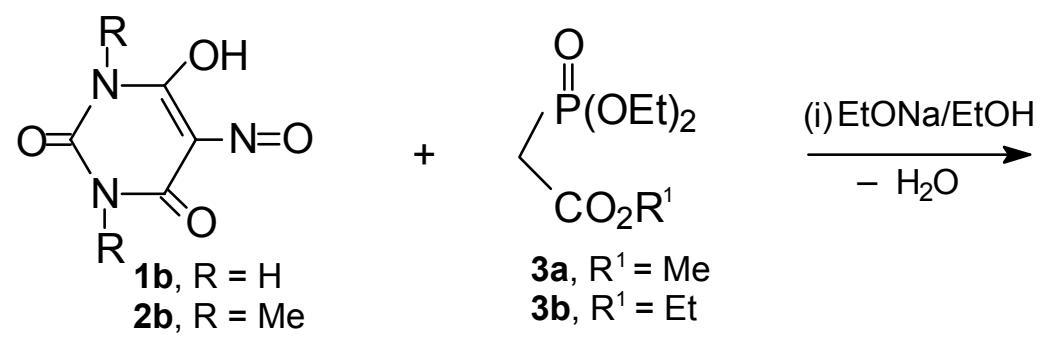<smiles>[R20]O[R12](=O)c1nc2c(=O)n([R])c(=O)n([R])c2oc1=O</smiles>

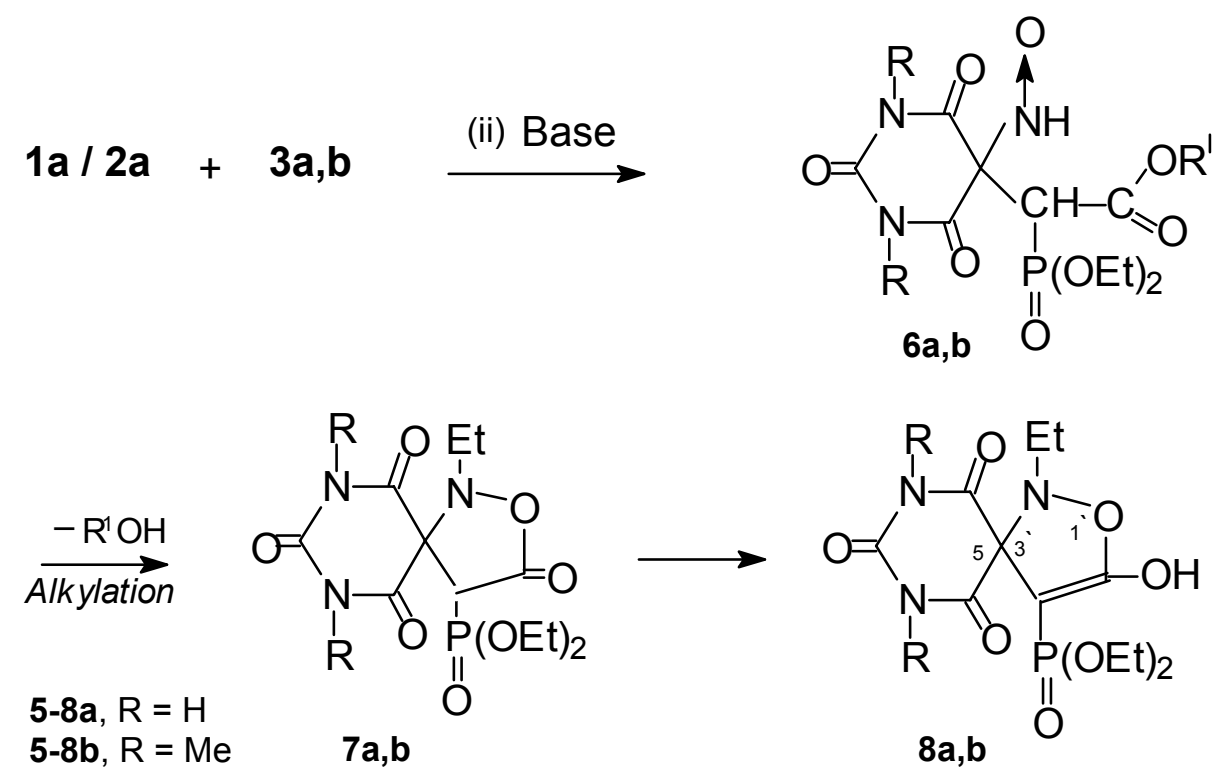

\section{Scheme 2}

Compound 5a $(\delta p=24.3 \mathrm{ppm})$ showed in its ${ }^{1} \mathrm{H}$ NMR spectrum $\left(\mathrm{d}_{6}\right.$-DMSO) the ethyl group of the phosphonate ester at $\delta 0.98,1.21\left(2 \mathrm{dt}, J_{H H}=6.5, J_{P H}=3.8 \mathrm{~Hz}, 2 \times 3 \mathrm{H}, 2 \times H_{3}\right.$ C.C.O), and at $\delta$ 3.75-3.89 (2dq (m), 4H, $\left.2 \times \mathrm{H}_{2} \mathrm{CO}\right)$. Its ${ }^{13} \mathrm{C}$ NMR spectrum exhibited two signals of the ethoxy moiety at $\delta c 15.9$ and 62.3. Other signals were observed at $\delta c 161.6\left(\mathrm{~d},{ }^{1} J_{P C}=200 \mathrm{~Hz}\right.$, $C(3)-\mathrm{P}), 152.4,161.2$ (5- and 7-C $=\mathrm{O}$ ) and $144.7\left(\mathrm{~d},{ }^{2} J_{P C}=28 \mathrm{~Hz}, 2-C=\mathrm{O}\right.$, lactone). The mass spectrum of 5a, as expected, confirmed the molecular weight. Initial fragmentations involved the loss of the side phosphonate species. The Main features of the IR-spectrum of $\mathbf{5 a}\left(\mathrm{in} \mathrm{KBr}, \mathrm{cm}^{-1}\right.$ ) were the presence of absorption bands at 1715, 1684, 1646 corresponding to 2-, 7, 5-carbonyl groups, respectively, $1262(\mathrm{P}=\mathrm{O}), 1073$ (P-O-C), and NH stretching frequencies at 3330 (br.).

The IR $\left(\mathrm{KBr}, \mathrm{cm}^{-1}\right)$ spectrum of $\mathbf{8 b}$ exhibited the presence of three carbonyl stretching vibration bands at 1705,1685 , and 1664 corresponding to 2,4,6-carbonyl groups of pyrimidine, 
thus excluding any cyclization reaction including these moieties. Other bands appeared at $v_{\max }$ $\left(\mathrm{cm}^{-1}\right)$ : $3410(\mathrm{OH}), 1238\left(\mathrm{P}=\mathrm{O}\right.$, bonded), and at $1080(\mathrm{P}-\mathrm{O}-\mathrm{C})$. The ${ }^{1} \mathrm{H}$ NMR spectrum $\left(\mathrm{CDCl}_{3}\right)$ of $\mathbf{8 b}(\delta p=27.6 \mathrm{ppm})$ displayed signals at $0.97,1.18\left(2 \mathrm{dt}, J_{H H}=6.7, J_{P H}=4 \mathrm{~Hz}, 2 \times 3 \mathrm{H}\right.$, $2 H_{3}$ C.C.O), and at $\delta 3.85,4.08\left(2 \mathrm{dq}, J_{H H}=6.7, J_{H P}=4.5 \mathrm{~Hz}, 4 \mathrm{H}, 2 \mathrm{H}_{2} \mathrm{CO}\right)$ due to the phosphonate species $\left[\mathrm{P}\left(\mathrm{OC}_{2} \mathrm{H}_{5}\right)_{2}\right]$ whereas the $N$-ethyl moiety was located at $\delta 0.87\left(\mathrm{t}, J_{H H}=7.4\right.$ $\mathrm{Hz}, 3 \mathrm{H}, H_{3}$ C.C.N) and at $\delta 4.43\left(\mathrm{q}, J_{H H}=7.4 \mathrm{~Hz}, 2 \mathrm{H}, H_{2} \mathrm{C}-\mathrm{N}\right)$. 1- and 3-N-methyl protons gave two singlets at 3.12, 3.27 and the 5'-hydroxyl proton resonated at 12.11 . Its ${ }^{13} \mathrm{C} \mathrm{NMR}$ spectrum displayed the phosphonate-carbon atom ( $4^{\circ} \mathrm{C}$-P) signal at $103.7\left(\mathrm{~d},{ }^{1} J_{P_{-} C}=207 \mathrm{~Hz}\right)$. Other two signals were observed at $\delta 67.8\left(\mathrm{~d},{ }^{2} J_{P_{-} C}=33 \mathrm{~Hz}\right), 180.3\left(\mathrm{~d},{ }^{2} J_{P_{-} C}=38 \mathrm{~Hz}\right)$ assignable to spiro$3^{\prime} \mathrm{C}$ and $\left.5^{\prime}-\mathrm{C}-\mathrm{OH}\right)$, respectively. These data indicate that the initially formed products 7 undergo prototropic rearrangement to the enolic form $\mathbf{8}$.

According to Scheme 2, carbanions 3 readily condensed with $\mathbf{1} / \mathbf{2}$ in the tautomeric nitrosa form to give the intermediates 4 (Perkin-type condensation). ${ }^{5}$ Perkin-type reaction was previously described for the reaction of $\mathbf{3}$ with benzaldehyde. ${ }^{6}$ Intramolecular cyclization of $\mathbf{4}$ with concomitant extrusion of an alcohol molecule afforded the substituted-oxazine phosphor esters 5. On the other hand, formation of $\mathbf{8}$ might involve an initial nucleophilic attack by the phosphonyl carbanions $\mathbf{3}$ on 2-hydroxyimino carbon $(2-C=\mathrm{NOH})$ in $\mathbf{1}$ and $\mathbf{2}^{7}$ yielding the phosphonates 6. Subsequent ring closure the spiro products $\mathbf{8}$ would be obtained via the intermediates 7. Under elimination of an appropriate alcohol moiety from 6 with concomitant $N$ alkylation the intermediates 7 are presumably formed. Considering the $N$-alkylation by WHreagent, an analogous process has been observed in the reactions of WH synthons with pyrroles, ${ }^{8 \mathrm{a}}$ quinonimines, ${ }^{8 \mathrm{~b}}$ nitrosonaphthol ${ }^{8 \mathrm{c}}$ and pyrimidines. ${ }^{8 \mathrm{~d}}$

In a systematic study, treatment of $\mathbf{1}$ and $\mathbf{2}$ with diethyl cyanomethylphosphonate (9) afforded diethyl (2-amino-5,7-dioxo-5,6,7,8-tetrahydropyrimidino[4,5-b][1,4]oxazin-3yl)phosphonate (10a, 68\%) [or diethyl (7-amino-6,8-dimethyl-5,7-dioxo-5H, 7H-pyrimidino[4,5b][1,4]-oxazin-3yl)phosphonate (10b), 74\%], via the intermediate $\mathbf{4 A}$, as the only identifiable product (Scheme 3$)$. The ${ }^{1} \mathrm{H}$ NMR spectrum of $\mathbf{1 0 b}\left(\delta_{P}=23.5 \mathrm{ppm}\right)$ showed two types of the $\mathrm{NH}_{2}$-protons $\left[\delta\left(H^{a}\right)=6.75(\mathrm{~s}, \mathrm{br}, 1 \mathrm{H})\right.$ and $\left.\delta\left(H^{\mathrm{b}}\right)=9.78(\mathrm{~s}, \mathrm{br}, 1 \mathrm{H})\right]$. The different chemical shifts of the $\mathrm{NH}_{2}$-protons are the spectroscopic evidence for the presence of intramolecular hydrogen bond between one of the hydrogens of the $\mathrm{NH}_{2}$-protons and the oxygen atom of the $\mathrm{P}=\mathrm{O}$ bonding in the phosphonate group. The $C(3)$ atom appeared as a doublet at $98.3\left({ }^{1} J_{P-C}=211\right.$ $\mathrm{Hz}$ ) in the ${ }^{13} \mathrm{C}$ NMR spectrum of $\mathbf{1 0 b}$. 
<smiles>[R]n1c(O)c(N=O)c(=O)n([R])c1=O</smiles>

1b, $R=H$

2b, $\mathrm{R}=\mathrm{Me}$<smiles>CCO[Pb](=O)CC#N</smiles>

9

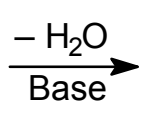<smiles>[R]O[P+](=O)/C(C#N)=N/c1c(O)n([R])c(=O)n([R])c1=O</smiles>

4A

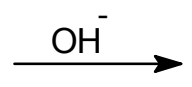

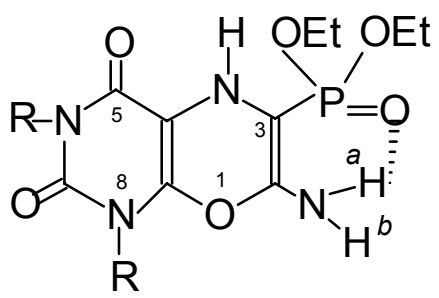

10a, $R=H ; 10 b, R=M e$

\section{Scheme 3}

Fused oxazines 10 were formed most probably through cyclization and transformation of the cyano group of initially formed condensation intermediates 4A. Similar observation was previously reported by Coppola et al ${ }^{9}$ for the reaction product of $N$-methyl isatoic anhydride with WH 9. Nevertheless, it should be noted that the observed behavior of $\mathbf{9}$ toward $\mathbf{1}$ is in marked disparity with the behavior of the phosphorane counterpart, cyanomethylenetriphenylphosphorane toward oxime 1. In the latter case, cycloaddition was observed, followed by an extrusion of a water molecule to give fused-2-cyano-1,3-oxazole $\mathbf{E}\left(\mathrm{R}^{2}=\mathrm{CN}\right)$ (Scheme 1). ${ }^{1}$

Next, we studied the reactions of $\mathbf{1}$ and $\mathbf{2}$ with diethyl ( $\alpha$-alkylthiomethyl)phosphonates 11 a and 11b. By a similar treatment of oxime 1 with diethyl ( $\alpha$-methylthiomethyl)phosphonate (11a) diethyl (3-ethoxy-4,5,6,7-tetrahydroxazolo[5,4- $d$ ]pyrimidine-4,6-dione)-2-phosphonate (13a, $74 \%$ ) was obtained as the sole reaction product according to Scheme 4 . The preferential extrusion of $\mathrm{R}^{2} \mathrm{SH}\left(\mathrm{R}^{2}=\mathrm{Me}\right.$ or Et, as it is monitored by its characteristic smell) than $\mathrm{HOH}$ molecule ${ }^{10}$ was driven from the result of the reaction of $\mathbf{1}$ with diethyl ( $\alpha$-ethylthiomethyl)phosphonate (11b). When 1 was caused to react with 11b, the oxazole 13a (76\%) was again obtained. On the same ground, oxime 2 reacted with either 11a or $11 \mathbf{b}$ to give the corresponding fused substituted oxazole phosphor ester 13b $(\approx 83 \%)$ (Scheme 4$)$. 

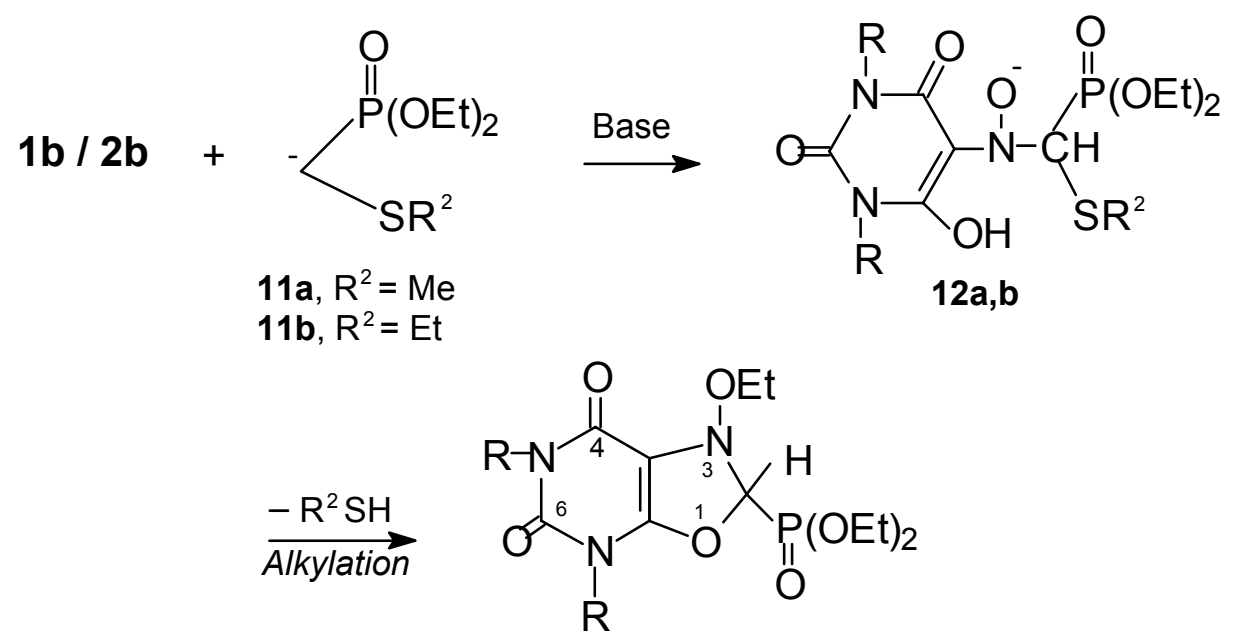

13a, $R=H ; 13 b, R=M e$

\section{Scheme 4}

The behavior of oximes 1 and $\mathbf{2}$ toward unsaturated phosphonyl carbanions $\mathbf{1 4}$ and $\mathbf{2 2}$ was then studied and the obtained products were predicted in Schemes 5 and 6. Treatment of 1 with diethyl vinylphosphonate (14) in ethyl alcohol in the presence of sodium ethanolate yielded diethyl (4-ethyl-5,7-dioxo-5,6,7,8-tetrahydropyrimidino[4,5-b][1,4]oxazin-3yl)-phosphonate (17a, 41\% yield) along with 1-ethoxy-4,5,6,7-tetrahydropyrrolo[4,5- $d$ ]pyrimidine -5,7-dione (21a, 32\% yield); or 6,8-dimethyl analogs $\mathbf{1 7 b}(43 \%)$ and $\mathbf{2 1 b}(26 \%)$ in the second reaction (with oxime 2). According to Scheme 5, the first step is the addition of the carbanion species $\mathbf{1 4}$ to $\mathbf{1 b}$ / $\mathbf{2 b}$ to give the intermediate $\mathbf{1 5}$ followed by intramolecular cyclization (under the elimination of a molecule of water) to yield the intermediate 16. Further $N$-alkylation afforded the products $\mathbf{1 7}$ (Scheme 5, route i). On the other hand, compounds 21 are regarded as products of an intramolecular Wittig-Horner reaction. Such an addition-cyclization product apparently results from an initial attack of the anions $\mathbf{A}$ on $\mathbf{1 4}$ forming the phosphonates 18, which transformed to 20 via phosphoryl species 19 elimination. Migration of the methine proton to the electron rich center accompanied with hydroxylamine alkylation would produce the final products 21 according to Scheme 5, route ii. ${ }^{11}$ Structure $\mathbf{2 1}$ is proved by elemental analysis and spectroscopic data as well as by analogy with compound $\mathbf{D}$ (Scheme 1), previously obtained from the reaction of 1 with vinyltriphenylphosphonium salt. ${ }^{1}$ 


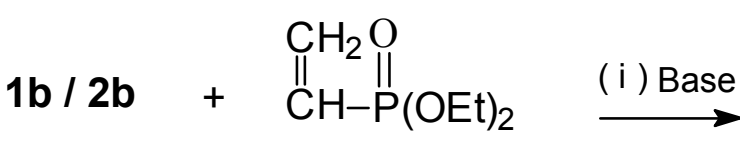

14<smiles>[R]n1c(OCC)c([N-][O-])c(=O)n([R])c1=O</smiles><smiles>[R]n1c(=O)c2c(c(=O)n1[R])OC=C(P(=O)(OCC)OCC)N2</smiles><smiles>[R]O[R](=O)c1coc2c([R])c(=O)n([R])c(=O)n12</smiles>

$17 a, R=H ; b, R=M e$

$1 / 2$<smiles>[R]N1C(=O)C(=N[O-])C(=O)N([R])C1=O</smiles>

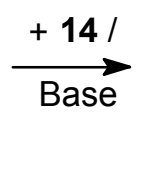

$1 A, R=H ; 2 A, R=M e$<smiles>[R]N1C(=O)C(=[N+]([O-])CP(=O)=O)C(=O)N([R])C1=O</smiles>

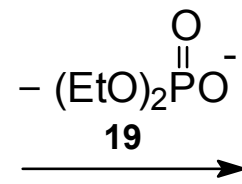

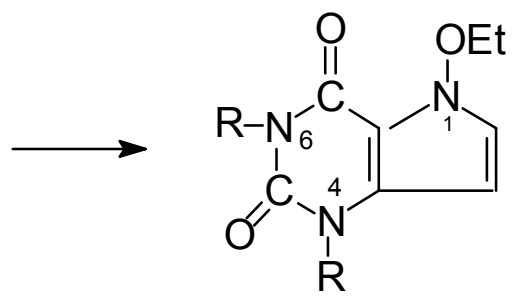

21a, $R=H ; b, R=M e$

\section{Scheme 5}

Next, when oxime 1 (or 2) was caused to react, under the same reaction conditions, with diethyl allylphosphonate (22) diethyl (5'-methyl-2,4,6-trioxo-1,2,3,4,6-pentahydrospiro[pyrimidine[5,3'][1,2]oxazol]-4'yl)phosphonate (25a, 34\% yield) and diethyl 2(3-ethyl-4,6dioxo-4,5,6,7-tetrahydro-1,3-oxazolo[5,4- $d]$ pyrimidine-2(1H)-ylidene)ethylphosphonate) (27a, $32 \%$ yield); or dimethylpyrimidine analogs $\mathbf{2 5 b}$ and $\mathbf{2 7 b}$ in equal yields $(\approx 35 \%$ ) were the reaction products (Scheme 6). Similar to $\mathbf{2 5}$ and $\mathbf{2 7}$, unphosphorylated oxazoles were previously ${ }^{1}$ obtained from the reaction of $\mathbf{1}$ with allyltriphenylphosphonium bromide (see Scheme 1).

Furthermore, the mechanisms outlined in Schemes 5 and 6 show a similar initial attack for the phosphonyl carbanions $\mathbf{1 4}$ and $\mathbf{2 2}$ and their phosphorane counterparts. ${ }^{1}$ However, further transformations are quite different. The main difference between the present reaction and the 
corresponding one of the Wittig reagent with the same substrate $\mathbf{1}^{1}$ is that, in the latter case, the formation of the products is accompanied by elimination of the phosphorus moiety $\left(\mathrm{Ph}_{3} \mathrm{P}\right)$. The contrasting behavior of the initial intermediate through elimination "of the phosphorus moiety" is because $\mathrm{Ph}_{3} \mathrm{P}$ is a much better leaving group than $\left[(E t O)_{2} \mathrm{PO}{ }^{-}\right]$moiety. There is much precedence for this difference. ${ }^{12}$ The results clearly show that oximes $\mathbf{1}$ and $\mathbf{2}$ react with phosphorus carbanions mainly in the nitrosa form, and not in the tautomeric hydroxyimino structure.

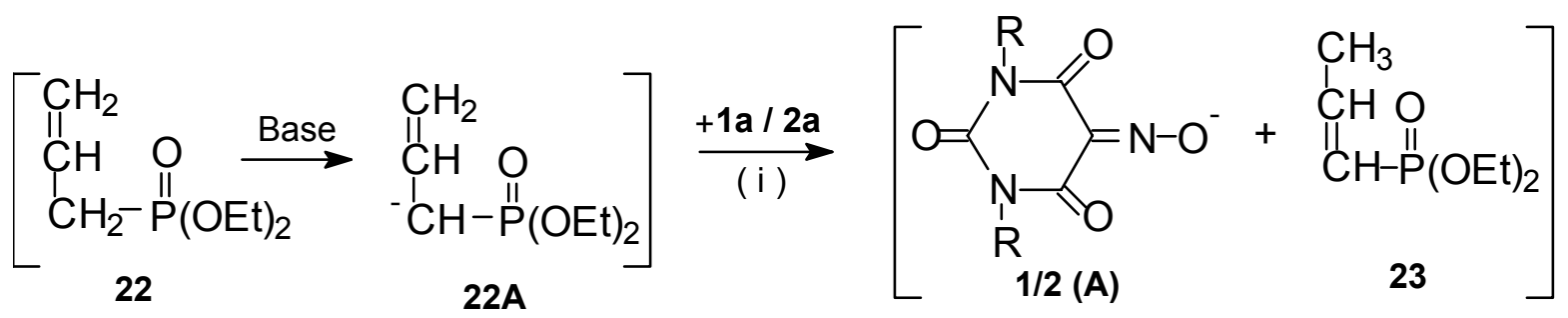

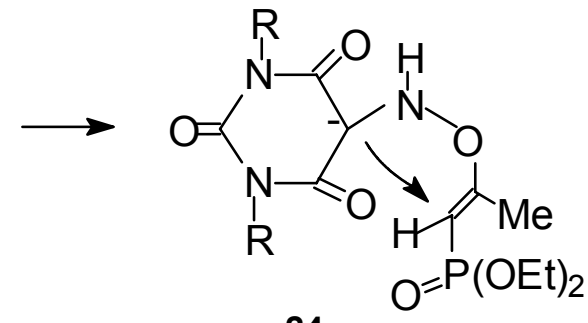

24

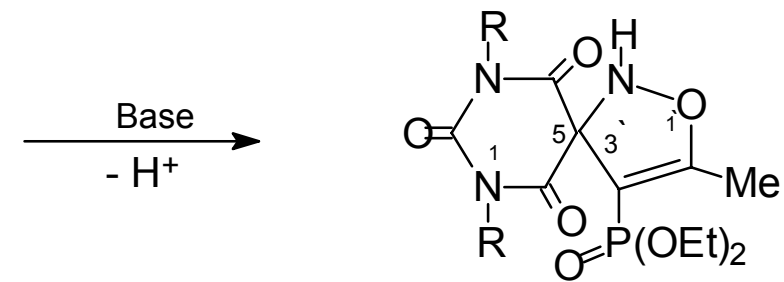

25a, $R=H ; b, R=M e$<smiles>[R]O[R10]#P</smiles>

\section{Scheme 6}

The structures suggested for all new compounds are in good agreement with their analytical and spectral data (Tables 1 and 2). 
Tabel 1. Physical properties, MS and spectral data for the products $\mathbf{5 a}, \mathbf{b}, \mathbf{8 a}, \mathbf{b}, \mathbf{1 0 a}, \mathbf{b}, \mathbf{1 3 a}, \mathbf{b}$, 17a,b, 21a,b, 25a,b, and 27a,b

\begin{tabular}{|c|c|c|c|c|c|c|c|c|}
\hline \multirow{3}{*}{$\begin{array}{l}\mathrm{Cmpd}^{\mathrm{a}} / \\
\text { Color }\end{array}$} & \multirow{3}{*}{$\begin{array}{l}\text { m.p. }\left({ }^{\circ} \mathrm{C}\right) \\
\text { solvent }\end{array}$} & \multirow{3}{*}{$\begin{array}{l}\text { Yield } \\
(\%)\end{array}$} & \multirow{3}{*}{$\begin{array}{l}\text { Mol. Form. (M. } \\
\text { Wt.) }\end{array}$} & \multicolumn{4}{|c|}{ Elemental Analysis } & \multirow{3}{*}{$\begin{array}{c}\text { MS: } m / z(\%)=\left[\mathrm{M}^{+}\right] \text {and relevant } \\
\text { fragments }\end{array}$} \\
\hline & & & & \multicolumn{4}{|c|}{ Calc. / Found } & \\
\hline & & & & $\mathrm{C} \%$ & $\mathrm{H} \%$ & $\mathrm{~N} \%$ & $\mathrm{P} \%$ & \\
\hline 5a/Straw & $240-241$ & $\approx 35^{\mathrm{b}}$ & $\mathrm{C}_{10} \mathrm{H}_{12} \mathrm{~N}_{3} \mathrm{O}_{7} \mathrm{P}$ & 37.87 & 3.81 & 13.25 & 9.76 & $317(8)\left[\mathrm{M}^{+}\right], 315(20), 301$ \\
\hline Yellow & $(\mathrm{MeOH})$ & & $(317.2)$ & 37.91 & 3.74 & 13.22 & 9.81 & $\begin{array}{l}\text { (100), } 164(63), 137(30), 108 \\
(55) .\end{array}$ \\
\hline $\mathbf{5 b} /$ Straw & $163-165$ & $\approx 38^{\mathrm{b}}$ & $\mathrm{C}_{12} \mathrm{H}_{16} \mathrm{~N}_{3} \mathrm{O}_{7} \mathrm{P}$ & 41.75 & 4.67 & 12.17 & 8.97 & $345(7)\left[\mathrm{M}^{+}\right], 330(23), 315(27)$, \\
\hline Yellow & $(\mathrm{MeCN})$ & & $(345.18)$ & 41.79 & 4.63 & 12.09 & 9.03 & $\begin{array}{l}301(100), 164(58), 137(34), \\
108(62) .\end{array}$ \\
\hline 8a / Straw & $212-215$ & $\approx 28^{\mathrm{b}}$ & $\mathrm{C}_{12} \mathrm{H}_{18} \mathrm{~N}_{3} \mathrm{O}_{8} \mathrm{P}$ & 39.68 & 4.99 & 11.57 & 8.53 & $363(12)\left[\mathrm{M}^{+}\right], 361(15), 345(48)$ \\
\hline Yellow & $(\mathrm{EtOH})$ & & $(363.27)$ & 39.77 & 4.94 & 11.51 & 8.60 & $\begin{array}{l}300(43), 163(100), 137(36) \\
124(51) .\end{array}$ \\
\hline $8 \mathbf{b} /$ & $158-160$ & $\approx 29^{\mathrm{b}}$ & $\mathrm{C}_{14} \mathrm{H}_{22} \mathrm{~N}_{3} \mathrm{O}_{8} \mathrm{P}$ & 42.97 & 5.66 & 10.74 & 7.91 & $391(27)\left[\mathrm{M}^{+}\right], 346(38), 331$ \\
\hline Orange & $\left(\mathrm{CHCl}_{3}\right)$ & & $(391.33)$ & 43.03 & 5.61 & 10.71 & 7.98 & $\begin{array}{l}(26), 316(72), 300(38), 163 \\
(100), 137(68), 124,124(53) .\end{array}$ \\
\hline $10 \mathrm{a} /$ & $266-268$ & 68 & $\mathrm{C}_{10} \mathrm{H}_{15} \mathrm{~N}_{4} \mathrm{O}_{6} \mathrm{P}$ & 37.74 & 4.75 & 17.61 & 9.73 & $318(13)\left[\mathrm{M}^{+}\right], 316(26), 299$ \\
\hline Colorless & $(\mathrm{EtOH})$ & & $(318.23)$ & 37.69 & 4.73 & 17.56 & 9.66 & $\begin{array}{l}(37), 162(77), 126(100), 108 \\
(60) .\end{array}$ \\
\hline $10 \mathbf{b} /$ & $171-172$ & 74 & $\mathrm{C}_{12} \mathrm{H}_{19} \mathrm{~N}_{4} \mathrm{O}_{6} \mathrm{P}$ & 41.62 & 5.53 & 16.18 & 8.94 & $346(29)\left[\mathrm{M}^{+}\right], 331(34), 316(52)$ \\
\hline Colorless & $\left(\mathrm{CH}_{2} \mathrm{Cl}_{2}\right)$ & & (346.28) & 41.67 & 5.57 & 16.19 & 8.86 & $\begin{array}{l}299(38), 162(72), 162(100) \\
108(66) .\end{array}$ \\
\hline 13a/Straw & $182-184$ & $\approx 75^{\mathrm{b}}$ & $\mathrm{C}_{11} \mathrm{H}_{18} \mathrm{~N}_{3} \mathrm{O}_{6} \mathrm{P}$ & 41.38 & 5.68 & 13.16 & 9.70 & $319(20)\left[\mathrm{M}^{+}\right], 317(36), 287(55)$ \\
\hline Yellow & $(\mathrm{MeCN})$ & & (319.26) & 41.43 & 5.64 & 13.08 & 9.77 & $137(28), 150(100), 108(72)$ \\
\hline $13 \mathbf{b} /$ & $122-123$ & $\approx 83^{\mathrm{b}}$ & $\mathrm{C}_{13} \mathrm{H}_{22} \mathrm{~N}_{3} \mathrm{O}_{6} \mathrm{P}$ & 44.95 & 6.38 & 12.10 & 8.92 & $347(9)\left[\mathrm{M}^{+}\right], 317$ (21), 287 (38), \\
\hline Yellow & $\left(\mathrm{CH}_{2} \mathrm{Cl}_{2}\right)$ & & $(347.32)$ & 45.02 & 6.31 & 12.18 & 8.99 & $137(35), 150$ (100), 108 (55). \\
\hline $17 \mathbf{a} /$ & 203-205 & 41 & $\mathrm{C}_{12} \mathrm{H}_{18} \mathrm{~N}_{3} \mathrm{O}_{6} \mathrm{P}$ & 43.51 & 5.48 & 12.68 & 9.35 & $331(13)\left[\mathrm{M}^{+}\right], 329(16), 300(65)$ \\
\hline Yellow & $(\mathrm{EtOH})$ & & $(331.27)$ & 43.48 & 5.44 & 12.73 & 9.41 & $\begin{array}{l}163(100), 149(61), 147(41) \\
108(50)\end{array}$ \\
\hline $17 \mathrm{~b} /$ & $170-173$ & 43 & $\mathrm{C}_{14} \mathrm{H}_{22} \mathrm{~N}_{3} \mathrm{O}_{6} \mathrm{P}$ & 46.80 & 6.17 & 11.69 & 8.62 & $359(12)\left[\mathrm{M}^{+}\right], 344(18), 329$ \\
\hline Yellow & (Benzene) & & $(359.32)$ & 46.73 & 6.16 & 11.64 & 9.63 & $\begin{array}{l}(24), 300(56), 163(100), 149 \\
(56), 147(33), 108(42) .\end{array}$ \\
\hline $21 \mathbf{a} /$ & 268-270 (iso- & 32 & $\mathrm{C}_{8} \mathrm{H}_{9} \mathrm{~N}_{3} \mathrm{O}_{3}$ & 49.23 & 4.65 & 21.53 & ---- & $195(14)\left[\mathrm{M}^{+}\right], 193(25), 148$ \\
\hline Colorless & $\mathrm{PrOH})$ & & $(195.18)$ & 49.26 & 4.59 & 21.55 & & $(100)$ \\
\hline $21 b /$ & 116-118 & 26 & $\mathrm{C}_{10} \mathrm{H}_{13} \mathrm{~N}_{3} \mathrm{O}_{3}$ & 53.80 & 5.87 & 18.82 & ----- & $223(21)\left[\mathrm{M}^{+}\right], 208(19), 193$ \\
\hline Colorless & Cyclohexane & & $(223.24)$ & 53.88 & 5.83 & 18.76 & & $(49), 148(100)$ \\
\hline $25 \mathbf{a} /$ & 193-195 & 34 & $\mathrm{C}_{11} \mathrm{H}_{16} \mathrm{~N}_{3} \mathrm{O}_{7} \mathrm{P}$ & 39.65 & 4.84 & 12.61 & 9.29 & $333(11)\left[\mathrm{M}^{+}\right], 331(28), 330$ \\
\hline Yellow & iso-PrOH & & $(333.24)$ & 39.61 & 4.89 & 12.55 & 9.21 & $\begin{array}{l}(21), 315(35), 178(100), 154 \\
(88), 124(65) .\end{array}$ \\
\hline $25 \mathbf{b} /$ & $151-152$ & 35 & $\mathrm{C}_{13} \mathrm{H}_{20} \mathrm{~N}_{3} \mathrm{O}_{7} \mathrm{P}$ & 43.22 & 5.58 & 11.63 & 8.57 & $361(9)\left[\mathrm{M}^{+}\right], 360(14), 345(16)$ \\
\hline Orange & $\left(\mathrm{CH}_{2} \mathrm{Cl}_{2}\right)$ & & (361.3) & 43.17 & 5.56 & 11.55 & 8.66 & $\begin{array}{l}330(31), 315(48), 178(100) \\
154(33), 124(60)\end{array}$ \\
\hline 27a / & $216-218$ & 32 & $\mathrm{C}_{13} \mathrm{H}_{20} \mathrm{~N}_{3} \mathrm{O}_{6} \mathrm{P}$ & 45.22 & 5.84 & 12.17 & 8.97 & $345(13)\left[\mathrm{M}^{+}\right], 343(29), 314(26)$, \\
\hline Yellow & $\left(\mathrm{CHCl}_{3}\right)$ & & $(345.3)$ & 45.25 & 5.77 & 12.09 & 9.04 & $164(58), 150(100), 108(82)$ \\
\hline 27b / & $154-156$ & 36 & $\mathrm{C}_{15} \mathrm{H}_{24} \mathrm{~N}_{3} \mathrm{O}_{6} \mathrm{P}$ & 48.26 & 6.48 & 11.26 & 8.30 & $373(15)\left[\mathrm{M}^{+}\right], 358(13), 343(39)$ \\
\hline Orange & $\mathrm{MeCN}$ & & $(373.35)$ & 48.29 & 5.52 & 11.32 & 8.26 & $\begin{array}{l}314(62), 164(62), 150(100), \\
108(66) .\end{array}$ \\
\hline
\end{tabular}

${ }^{\mathrm{a}}$ For further details, see the experimental section; ${ }^{\mathrm{b}}$ Average yield from the two experiments. 
Table 2. IR and ${ }^{1} \mathrm{H}-,{ }^{31} \mathrm{P}$ - and ${ }^{13} \mathrm{C}$ NMR Spectral data ${ }^{\mathrm{a}}$ for the products $\mathbf{5 a}, \mathbf{b}, \mathbf{8 a}, \mathbf{b}, \mathbf{1 0 a}, \mathbf{b}, \mathbf{1 3 a}, \mathbf{b}$, $17 \mathbf{a}, \mathbf{b}, 21 \mathbf{a}, \mathbf{b}, 25 \mathbf{a}, \mathbf{b}$, and $27 \mathbf{a}, \mathbf{b}$

\begin{tabular}{|c|c|c|c|}
\hline $\begin{array}{c}\text { Cmpd. } \\
\text { No. }\end{array}$ & $\begin{array}{c}\mathrm{IR}(\mathrm{KBr}),{ }^{\mathrm{a}} v_{\max } \\
\left(\mathrm{cm}^{-1}\right)\end{array}$ & ${ }^{1} \mathrm{H}-$ and ${ }^{31} \mathrm{P}$ NMR $\delta,(\mathrm{ppm})$ & ${ }^{13} \mathrm{C}$ NMR $\delta,(\mathrm{ppm})$ \\
\hline $5 a^{a}{ }^{a}$ & $\begin{array}{l}3330 \mathrm{w}(\mathrm{NHs}) \\
1715,1684 \\
1646(2,7,5- \\
\mathrm{C}=\mathrm{O}), 1262 \\
(\mathrm{P}=\mathrm{O}), 1073(\mathrm{P}- \\
\mathrm{O}-\mathrm{C})\end{array}$ & $\begin{array}{l}0.98,1.21\left(2 \mathrm{dt}, J_{H H}=6.5, J_{P H}=3.8 \mathrm{~Hz}, 6 \mathrm{H},\right. \\
\left.2 \times H_{3} \mathrm{CC} . \mathrm{O}\right), 3.75-3.89(2 \mathrm{qt}(\mathrm{m}), 4 \mathrm{H}, \\
\left.2 H_{2} \mathrm{CO}\right), 9.35,10.45(2 \mathrm{~s}, 2 \mathrm{H}, 2 \mathrm{NH}) . \\
\delta_{P}=24.3 \mathrm{ppm}\end{array}$ & $\begin{array}{l}15.9\left(C \mathrm{H}_{3} \mathrm{C} . \mathrm{O}\right), 62.3\left(\mathrm{CH}_{2} \mathrm{O}\right), \\
144.7\left(\mathrm{~d},{ }^{2} J_{P C}=28 \mathrm{~Hz}, 2-\right. \\
C=\mathrm{O}), 152.4,161.2(5-, 7- \\
C=\mathrm{O}), 161.6\left(\mathrm{~d},{ }^{1} J_{P C}=200 \mathrm{~Hz},\right. \\
3-C) .\end{array}$ \\
\hline $\mathbf{5} \mathbf{b}^{\mathrm{b}}$ & $\begin{array}{l}1722,1685 \\
1656(2-, 7-, 5- \\
\mathrm{C}=\mathrm{O}), 1260 \\
(\mathrm{P}=\mathrm{O}), 1058(\mathrm{P}- \\
\mathrm{O}-\mathrm{C})\end{array}$ & $\begin{array}{l}0.98,1.18\left(2 \mathrm{dt}, J_{H H}=6.5, J_{P H}=3.8 \mathrm{~Hz}, 6 \mathrm{H},\right. \\
\left.2 \times H_{3} \mathrm{CC} . \mathrm{O}\right), 3.14,3.18(2 \mathrm{~s}, 2 \times 3 \mathrm{H}, 2 \times \\
\left.\mathrm{NCH}_{3}\right), 3.87-4.08\left(2 \mathrm{dq}(\mathrm{m}), 4 \mathrm{H}, 2 \mathrm{H}_{2} \mathrm{CO}\right) ; \delta_{P} \\
=22.8 \mathrm{ppm}\end{array}$ & $\begin{array}{l}15.8\left(\mathrm{CH}_{3} \mathrm{C} . \mathrm{O}\right), 28.4,28.8(6-, \\
\left.\text { 8- } C \mathrm{H}_{3} \mathrm{~N}\right), 62.3\left(C \mathrm{H}_{2} \mathrm{O}\right), \\
150.5\left(\mathrm{~d},{ }^{2} J_{P C}=33 \mathrm{~Hz}, 2-C=\mathrm{O}\right), \\
152.4,161.2(5-, 7-C=\mathrm{O}), \\
161.9\left(\mathrm{~d},{ }^{1} J_{P C}=201 \mathrm{~Hz}, 3-\mathrm{C}\right) .\end{array}$ \\
\hline $8 a^{a}$ & $\begin{array}{l}3410(\mathrm{OH}), \\
3350 \mathrm{w}(\mathrm{NHs}), \\
1700,1680, \\
1665(2-, 4-, 6- \\
\mathrm{C}=\mathrm{O}), 1230 \\
(\mathrm{P}=\mathrm{O}, \text { bonded }), \\
1085 \\
(\mathrm{P}-\mathrm{O}-\mathrm{C}) .\end{array}$ & $\begin{array}{l}0.85\left(\mathrm{t}, J_{H H}=7.4 \mathrm{~Hz}, 3 \mathrm{H}, H_{3} \mathrm{C} . \mathrm{C} . \mathrm{N}\right), 0.99, \\
1.17\left(2 \mathrm{dt}, J_{H H}=6.5, J_{P H}=3.8 \mathrm{~Hz}, 2 \times 3 \mathrm{H}, 2\right. \\
\left.\times H_{3} \mathrm{C} . \mathrm{C} . \mathrm{O}\right), 3.89,4.07\left(2 \mathrm{qt}, J_{P H}=11.5 \mathrm{~Hz},\right. \\
\left.4 \mathrm{H}, 2 \times H_{2} \mathrm{CO}\right), 4.46\left(\mathrm{q}, J_{H H}=6.7 \mathrm{~Hz}, 2 \mathrm{H},\right. \\
\left.\mathrm{N}-\mathrm{C} H_{2}\right), 9.55,10.65(2 \mathrm{~s}, 2 \times 1 \mathrm{H}, 1-, 3-\mathrm{N} H), \\
12.31\left(5^{`}-\mathrm{O} H\right) ; \delta_{P}=29.4 \mathrm{ppm} .\end{array}$ & $\begin{array}{l}14.4\left(\mathrm{CH}_{3} \mathrm{C} . \mathrm{N}\right), 15.4 \\
\left(\mathrm{CH}_{3} \mathrm{C} . \mathrm{O}\right), 43.6\left(\mathrm{CH}_{2} \mathrm{~N}\right), 61.6 \\
\left(\mathrm{CH}_{2} \mathrm{O}\right), 67.9\left(\mathrm{~d},{ }^{2} J_{P C}=33 \mathrm{~Hz},\right. \\
\left.3^{`}-\mathrm{C}-\text { spiro }\right), 102.5\left(\mathrm{~d},{ }^{1} J_{P C}=\right. \\
\left.213 \mathrm{~Hz}, 4^{`}-\mathrm{C}\right), 152.3,154.4(4- \\
, 2-, 6-C=\mathrm{O}), 178.9\left(\mathrm{~d},{ }^{2} J_{P C}=\right. \\
\left.38.2 \mathrm{~Hz}, 5^{`}-C-\mathrm{OH}\right) .\end{array}$ \\
\hline $\mathbf{8} \mathbf{b}^{\mathrm{b}}$ & $\begin{array}{l}3410(\mathrm{OH}), \\
1705,1685, \\
1664(2-, 4-, 6- \\
\mathrm{C}=\mathrm{O}), 1238 \\
(\mathrm{P}=\mathrm{O}, \text { bonded }) \\
1080 \\
(\mathrm{P}-\mathrm{O}-\mathrm{C})\end{array}$ & $\begin{array}{l}0.87\left(\mathrm{t}, J_{H H}=7.4 \mathrm{~Hz}, 3 \mathrm{H}, H_{3} \mathrm{C} . \mathrm{C} . \mathrm{N}\right), 0.97, \\
1.18\left(2 \mathrm{dt}, J_{H H}=6.7, J_{P H}=4 \mathrm{~Hz}, 6 \mathrm{H}, 2 \times\right. \\
\left.H_{3} \text { C.C.O }\right), 3.12,3.27(2 \mathrm{~s}, 2 \times 3 \mathrm{H}, 2 \mathrm{NCH}), \\
3.85,4.08\left(2 \mathrm{dq}, J_{H H}=6.7, J_{P H}=4.5 \mathrm{~Hz}, 2\right. \\
\left.\times 2 \mathrm{H}, 2 \times H_{2} \mathrm{CO}\right), 4.43\left(\mathrm{q}, J_{H H}=7.4 \mathrm{~Hz},\right. \\
\left.2 \mathrm{H}, \mathrm{NC} H_{2}\right), 12.11\left(\mathrm{~s}, 1 \mathrm{H}, 5{ }^{`}-\mathrm{OH}\right) ; \\
\delta_{P}=27.6 \mathrm{ppm} .\end{array}$ & $\begin{array}{l}14.6\left(\mathrm{CH}_{3} \mathrm{C} . \mathrm{N}\right), 15.5\left(\mathrm{CH}_{3} \mathrm{CO}\right), \\
27.8,28.1\left(1-, 3-\mathrm{NCH}_{3}\right), 44.3 \\
\left(\mathrm{CH}_{2} \mathrm{~N}\right), 61.7\left(\mathrm{OCH}_{2}\right), 67.8(\mathrm{~d}, \\
\left.{ }^{2} J_{P C}=33 \mathrm{~Hz}, 3^{`}-C \text {-spiro }\right), \\
103.7\left(\mathrm{~d},{ }^{1} J_{P C}=207 \mathrm{~Hz}, 4^{`}-C\right), \\
152.9,159.4(4-, 2-, 6-C=\mathrm{O}), \\
180.3\left(\mathrm{~d},{ }^{2} J_{P C}=38.4 \mathrm{~Hz}, 5^{`}-C-\right. \\
\mathrm{OH}) .\end{array}$ \\
\hline
\end{tabular}


$10 \mathrm{a}^{\mathrm{a}} \quad 3340 w\left(\mathrm{NHs}, \quad 0.98,1.11\left(2 \mathrm{dt}, J_{H H}=6.5, J_{P H}=3.8 \mathrm{~Hz}, 2 \times\right.\right.$ $\left.\mathrm{NH}_{2}\right), 1685$, $1645(7,5-$

$\mathrm{C}=\mathrm{O}), 1232$

$(\mathrm{P}=\mathrm{O}$, bonded $)$ 1077

(P-O-C).

$10 \mathrm{~b}$

$3330 w(\mathrm{NH}$

$\left.\mathrm{NH}_{2}\right), 1685$, $1645(7-, 5-$

$\mathrm{C}=\mathrm{O}), 1232$

$(\mathrm{P}=\mathrm{O}$, bonded $)$

1084

(P-O-C).

$13 a^{a}$

$3330_{\omega}(\mathrm{NH})$, $1685,1650(6-$, 4-C $=\mathrm{O}), 1257$ $(\mathrm{P}=\mathrm{O}), 1055$ (P$\mathrm{O}-\mathrm{C})$.

$\mathbf{1 3 b}^{\mathrm{b}} \quad 1680,1650(6-$, 4-C $=\mathrm{O}), 1255$ $(\mathrm{P}=\mathrm{O}), 1087$ (P-O-C).

$17 \mathbf{a}^{\mathrm{a}} \quad 3340$ (NHs), 1685,1648 (7-, 5-C $=\mathrm{O}), 1265$ $(\mathrm{P}=\mathrm{O}), 1064$ (P-O-C).

$17 b^{a}$

$$
3 \mathrm{H}, 2 \times H_{3} \text { CC.O), 3.88, } 4.12\left(2 \mathrm{dq}, J_{H H}=\right.
$$$$
\text { 6.5, } \left.J_{P H}=4.5 \mathrm{~Hz}, 2 \times 2 \mathrm{H}, 2 \times \mathrm{H}_{2} \mathrm{CO}\right), 6.47
$$

$\left(\mathrm{s}, \mathrm{br}, 1 \mathrm{H}, \mathrm{N} H^{a}\right), 9.89\left(\mathrm{~s}, \mathrm{br}, 1 \mathrm{H}, \mathrm{N} H^{b}\right), 9.56$, $9.77,10.67(3 \mathrm{~s}(\mathrm{br}), 3 \times 1 \mathrm{H}, 3 \times \mathrm{N} H) ; \delta_{P}=$ $21.6 \mathrm{ppm}$.

$0.95,1.16\left(2 \mathrm{dt}, J_{H H}=6.7, J_{P H}=4.12 \mathrm{~Hz}, 2\right.$ $\times 3 \mathrm{H}, 2 \times H_{3}$ C.C.O) $, 3.18,3.28(2 \mathrm{~s}, 6 \mathrm{H}, 2 \times$ $\mathrm{NCH}_{3}$ ), 3.89, 4.07 (2dq, $J_{H H}=6.7, J_{P H}=4.8$ $\left.\mathrm{Hz}, 2 \times 2 \mathrm{H}, 2 \times \mathrm{H}_{2} \mathrm{CO}\right), 6.75(\mathrm{~s}, \mathrm{br}, 1 \mathrm{H}$, $\left.\mathrm{N} H^{a}\right), 9.78\left(\mathrm{~s}, \mathrm{br}, 1 \mathrm{H}, \mathrm{N} H^{b}\right), 10.08(\mathrm{~s}, 1 \mathrm{H}, 4-$ $\mathrm{N} H) ; \delta_{P}=23.5 \mathrm{ppm}$.

\subsection{5-1.32 (3t (m), 9H, $H_{3}$ C.CON \& 2} $H_{3}$ C.C.O), 3.72, 3.99-4.23 (3q (m), 6H, $2 \times$ $\left.\mathrm{H}_{2} \mathrm{CO} \& \mathrm{H}_{2} \mathrm{CON}\right), 5.36\left(\mathrm{~d}, J_{P H}=18.6 \mathrm{~Hz}\right.$, $1 \mathrm{H}, H \mathrm{C}-\mathrm{P}), 8.88,9.71(2 \mathrm{~s}, 2 \times 1 \mathrm{H}, 2 H \mathrm{~N})$; $\delta_{P}=18.7 \mathrm{ppm}$.

$1.08-1.26\left(3 \mathrm{t}(\mathrm{m}), 9 \mathrm{H}, H_{3} \mathrm{C} . \mathrm{CON} \& 2 \times\right.$ $H_{3}$ C.C.O), 3.08, $3.23\left(2 \mathrm{~s}, 6 \mathrm{H}, 2 \mathrm{NCH}_{3}\right)$, 3.75, 3.99-4.31 (3q (m), 6H, $2 \times \mathrm{H}_{2} \mathrm{CO} \&$ $\left.H_{2} \mathrm{CON}\right),\left(\mathrm{d}, J_{P H}=20.1 \mathrm{~Hz}, 1 \mathrm{H}, H \mathrm{C}-\mathrm{P}\right)$, $\delta_{P}=18.3 \mathrm{ppm}$.

0.90-1.21 (3t(m), 9H, H 3 C.C-N \& $2 \times$ $\mathrm{H}_{3}$ C.C.O), 3.99-4.33 (3q (m), 6H, $2 \times \mathrm{H}_{2} \mathrm{CO}$ $\left.\& H_{2} \mathrm{C}-\mathrm{N}\right), 6.47\left(\mathrm{~d}, J_{P H}=4.4 \mathrm{~Hz}, 1 \mathrm{H}, 2-\right.$ $\mathrm{CH}), 9.63,10.45(2 \mathrm{~s}, 2 \times 1 \mathrm{H}, 2 H \mathrm{~N}) ; \delta_{P}=$ $26.1 \mathrm{ppm}$.

$1682,1638(7-$, $5-\mathrm{C}=\mathrm{O}), 1580$

$(\mathrm{C}=\mathrm{N}), 1268$

$(\mathrm{P}=\mathrm{O}), 1110$

(P-O-C).
$16.5\left(\mathrm{CH}_{3} \mathrm{C} . \mathrm{O}\right), 61.8\left(\mathrm{CH}_{2} \mathrm{O}\right)$, $96.3\left(\mathrm{~d},{ }^{1} J_{P C}=211 \mathrm{~Hz}, 3-C\right)$, $130.7\left(\mathrm{~d},{ }^{2} J_{P C}=37 \mathrm{~Hz}, 2-C\right)$, 154.5, $160.6(5-, 7-C=\mathrm{O})$.

16.5 ( $\mathrm{CH}_{3}$ C.O), 28.4, 28.8 (6-, 8- $\left.\mathrm{CH}_{3} \mathrm{~N}\right), 63.7\left(\mathrm{CH}_{2} \mathrm{O}\right), 98.3$ $\left(\mathrm{d},{ }^{1} J_{P C}=211 \mathrm{~Hz}, 3-C\right), 131.4$ $\left(\mathrm{d},{ }^{2} J_{P C}=37 \mathrm{~Hz}, 2-C\right), 154.8$, $161.6(5-, 7-C=\mathrm{O})$.

$16.1\left(\mathrm{CH}_{3}\right.$ C. O $), 62.2\left(\mathrm{CH}_{2} \mathrm{O}\right)$, $111.9\left(\mathrm{~d},{ }^{l} J_{P C}=222 \mathrm{~Hz}, 2-C\right)$, 152.4, $161.5(4-, 6-C=\mathrm{O})$.

$16.3\left(\mathrm{CH}_{3}\right.$ C. O), 27.8, 28.3 (5-, 7- $\left.\mathrm{CH}_{3} \mathrm{~N}\right), 53.7\left(\mathrm{CH}_{2} \mathrm{ON}\right), 62.3$ $\left(\mathrm{CH}_{2} \mathrm{O}\right), 114.8\left(\mathrm{~d},{ }^{1} J_{P C}=216\right.$ $\mathrm{Hz}, 2-C), 151.8,161.8$ (4-, 6$C=\mathrm{O})$.

$15.6\left(\mathrm{CH}_{3} \mathrm{C} . \mathrm{O}\right), 17.7$

$\left(\mathrm{CH}_{3} \mathrm{C} . \mathrm{N}\right), 44.8\left(\mathrm{CH}_{2} \mathrm{~N}\right), 61.6$ $\left(\mathrm{CH}_{2} \mathrm{O}\right), 102.7\left(\mathrm{~d},{ }^{l} J_{P C}=201\right.$ $\mathrm{Hz}, 3-C), 125.8\left(\mathrm{~d},{ }^{2} J_{P C}=38\right.$ $\mathrm{Hz}, 2-C), 155.8,161.2$ (5-, 7$C=\mathrm{O}$ ).

$15.4\left(\mathrm{CH}_{3} \mathrm{C} . \mathrm{O}\right), 17.9\left(\mathrm{CH}_{3} \mathrm{C}\right.$. N), 28.0, $28.8\left(6-, 8-\mathrm{CH}_{3} \mathrm{~N}\right)$, $43.8\left(\mathrm{CH}_{2} \mathrm{~N}\right), 61.5\left(\mathrm{CH}_{2} \mathrm{O}\right)$, $104.2\left(\mathrm{~d},{ }^{1} J_{P C}=179 \mathrm{~Hz}, 3-C\right)$, $126.4\left(\mathrm{~d},{ }^{2} J_{P C}=46 \mathrm{~Hz}, 2-C\right)$, 153.5, $161.3(5-, 7-C=O)$. 


\begin{tabular}{|c|c|c|c|}
\hline $21 \mathrm{a}^{\mathrm{a}}$ & $\begin{array}{l}3335 \mathrm{w}(\mathrm{NHs}) \\
1685,1645(5- \\
\text { and } 7-\mathrm{C}=\mathrm{O})\end{array}$ & $\begin{array}{l}1.40\left(\mathrm{t}, J_{H H}=6.9 \mathrm{~Hz}, H_{3} \mathrm{C} . \mathrm{C} . \mathrm{ON}\right), 4.77(\mathrm{q}, \\
\left.J_{H H}=6.9 \mathrm{~Hz}, 2 \mathrm{H}, H_{2} \mathrm{CO}-\mathrm{N}\right), 6.34,6.76(2 \mathrm{~d}, \\
\left.J_{H H}=2.8 \mathrm{~Hz}, 2 \mathrm{H}, 2-, 3-H \mathrm{C}\right), 9.63,10.45 \\
(2 \mathrm{~s}, 2 \times 1 \mathrm{H}, 2 \times H \mathrm{~N})\end{array}$ & $\begin{array}{l}15.4\left(\mathrm{CH}_{3} \mathrm{C} . \mathrm{ON}\right), 58.2\left(\mathrm{CH}_{2} \mathrm{O}-\right. \\
\mathrm{N}), 99.2(3-C), 138.9(2-C), \\
151.4,167.3(7-, 5-C=O) .\end{array}$ \\
\hline $21 b^{b}$ & $\begin{array}{l}1688,1637(7- \\
5-\mathrm{C}=\mathrm{O})\end{array}$ & $\begin{array}{l}1.42\left(\mathrm{t}, J_{H H}=6.9 \mathrm{~Hz}, H_{3} \text { C.C.ON }\right), 3.14 \\
3.19\left(\mathrm{~s}, 2 \times 3 \mathrm{H}, 2 \times \mathrm{NCH}_{3}\right), 4.73\left(\mathrm{q}, J_{H H}=\right. \\
\left.6.9 \mathrm{~Hz}, 2 \mathrm{H}, H_{2} \mathrm{C} . \mathrm{ON}\right), 6.32,6.51\left(2 \mathrm{~d}, J_{H H}=\right. \\
2.8 \mathrm{~Hz}, 2 \times 1 \mathrm{H}, 2-, 3-H \mathrm{C}) .\end{array}$ & $\begin{array}{l}15.6\left(\mathrm{CH}_{3} \mathrm{C} . \mathrm{ON}\right), 27.4,29.2 \\
\left(4-, 6-\mathrm{CH}_{3} \mathrm{~N}\right), 58.6\left(\mathrm{CH}_{2} \mathrm{ON}\right), \\
98.2(3-C), 138.4(2-C), 151.6, \\
157.9(7-, 5-C=O) .\end{array}$ \\
\hline $25 a^{a}$ & $\begin{array}{l}3350 \mathrm{w}(\mathrm{NHs}) \\
1700,1682 \\
1638(2-, 4-, 6- \\
\mathrm{C}=\mathrm{O}), 1265 \\
(\mathrm{P}=\mathrm{O}), 1075 \\
(\mathrm{P}-\mathrm{O}-\mathrm{C})\end{array}$ & $\begin{array}{l}0.98,1.16\left(2 \mathrm{dt}, 2 \times 3 \mathrm{H}, J_{H H}=6.5, J_{P H}=3.8\right. \\
\left.\mathrm{Hz}, 2 H_{3} \mathrm{C} . \mathrm{C} . \mathrm{O}\right), 2.57\left(\mathrm{~d}, J_{P H}=4.2 \mathrm{~Hz}, 3 \mathrm{H}\right. \\
5 \mathrm{CH}), 3.79,4.03\left(2 \mathrm{dq}, J_{H H}=6.5, J_{P H}=4.4\right. \\
\left.\mathrm{Hz}, 4 \mathrm{H}, 2 \times H_{2} \mathrm{CO}\right), 9.46,10.76,11.43(3 \mathrm{~s}, \\
3 \mathrm{H}, 3 H \mathrm{~N}) ; \delta_{P}=25.6 \mathrm{ppm} .\end{array}$ & $\begin{array}{l}16.3\left(\mathrm{CH}_{3} \mathrm{C} . \mathrm{O}\right), 17.6\left(5^{`}-\right. \\
\left.\mathrm{C}^{-} \mathrm{CH}_{3}\right), 62.2\left(\mathrm{CH}_{2} \mathrm{O}\right), 72.4(\mathrm{~d}, \\
\left.{ }^{2} J_{P C}=37 \mathrm{~Hz}, 3^{`}-C \text {-spiro }\right), \\
120.5\left(\mathrm{~d},{ }^{1} J_{P C}=221 \mathrm{~Hz}, 4^{`}-C\right), \\
150.1,158.8(2-, 4-, 6-C=\mathrm{O}) .\end{array}$ \\
\hline $25 b^{b}$ & $\begin{array}{l}3230(\mathrm{NH}), \\
1700,1682, \\
1638(2-, 4-, 6- \\
\mathrm{C}=\mathrm{O}), 1580 \\
(\mathrm{C}=\mathrm{N}), 1260 \\
(\mathrm{P}=\mathrm{O}), 1075 \\
(\mathrm{P}-\mathrm{O}-\mathrm{C}) .\end{array}$ & $\begin{array}{l}0.98,1.21\left(2 \mathrm{dt}, 2 \times 3 \mathrm{H}, J_{H H}=6.5, J_{P H}=3.8\right. \\
\left.\mathrm{Hz}, 2 \times H_{3} \text { C.C.O }\right), 2.57\left(\mathrm{~d}, J_{P H}=4.2 \mathrm{~Hz},\right. \\
\left.3 \mathrm{H}, 5 \mathrm{CH}_{3}\right), 2.98,3.15\left(\mathrm{~s}, 2 \times 3 \mathrm{H}, 2 \mathrm{NCH} H_{3},\right. \\
3.99,4.13\left(2 \mathrm{dq}, J_{H H}=6.5, J_{P H}=4.4 \mathrm{~Hz}, 2 \times\right. \\
\left.2 \mathrm{H}, 2 \times H_{2} \mathrm{CO}\right), 11.76(\mathrm{~s}, 1 \mathrm{H}, H \mathrm{~N}) ; \delta_{P}= \\
23.7 \mathrm{ppm} .\end{array}$ & $\begin{array}{l}16.3\left(\mathrm{CH}_{3} \mathrm{C} . \mathrm{O}\right), 17.7\left(5^{`}-\right. \\
\left.\mathrm{C} . \mathrm{CH}_{3}\right), 27.4,27.8\left(1-, 3^{-}\right. \\
\left.C_{3} \mathrm{~N}\right), 62.2\left(\mathrm{CH}_{2} \mathrm{O}\right), 71.7(\mathrm{~d}, \\
\left.{ }^{2} J_{P C}=37 \mathrm{~Hz}, 3^{`}-C-\text { spiro }\right), \\
121.0\left(\mathrm{~d},{ }^{1} J_{P C}=218 \mathrm{~Hz}, 4^{`}-\mathrm{C}\right), \\
150.8,170.3(2-, 4-, 6-C=\mathrm{O}) .\end{array}$ \\
\hline $27 a^{a}$ & $\begin{array}{l}3350 \mathrm{w}(\mathrm{NHs}) \\
1688,1641(6-, \\
4-\mathrm{C}=\mathrm{O}), 1265 \\
(\mathrm{P}=\mathrm{O}), 1048 \\
(\mathrm{P}-\mathrm{O}-\mathrm{C})\end{array}$ & $\begin{array}{l}0.90-1.23\left(3 \mathrm{t}(\mathrm{m}), 9 \mathrm{H}, H_{3} \mathrm{C} . \mathrm{C}-\mathrm{N} \& 2 \times\right. \\
\left.H_{3} \mathrm{C} . \mathrm{C} . \mathrm{O}\right), 2.45\left(\mathrm{dd}, J_{H H}=7,{ }^{2} J_{P H}=15.8 \mathrm{~Hz},\right. \\
\left.2 \mathrm{H}, H_{2} \mathrm{C}-\mathrm{P}\right), 3.99-4.32(3 \mathrm{q}(\mathrm{m}), 3 \times 2 \mathrm{H}, 2 \times \\
\left.H_{2} \mathrm{CO} \& H_{2} \mathrm{C}-\mathrm{N}\right), 4.97\left(\mathrm{dt}, J_{H H}=7,{ }^{3} J_{P H}=\right. \\
4.4 \mathrm{~Hz}, 1 \mathrm{H},=\mathrm{C} H), 9.16,10.58(2 \mathrm{~s}, 2 \mathrm{H}, \\
2 H \mathrm{~N}) ; \quad \delta_{P}=20.4 \mathrm{ppm} .\end{array}$ & $\begin{array}{l}14.4\left(\mathrm{CH}_{3} \mathrm{C} . \mathrm{N}\right), 16.3 \\
\left(\mathrm{CH}_{3} \mathrm{C} . \mathrm{O}\right), 27.5\left(\mathrm{~d},{ }^{l} J_{P C}=111\right. \\
\left.\mathrm{Hz},-C \mathrm{H}_{2} \mathrm{P}\right), 43.6\left(C \mathrm{H}_{2} \mathrm{~N}\right), 62.9 \\
\left(C \mathrm{H}_{2} \mathrm{O}\right), 65.7(=C \mathrm{H}), 140.3(2- \\
C), 154.6,162.4(4-, 6-C=\mathrm{O}) .\end{array}$ \\
\hline $27 b^{b}$ & $\begin{array}{l}1682,1638(6- \\
4-\mathrm{C}=\mathrm{O}), 1265 \\
(\mathrm{P}=\mathrm{O}), 1056 \\
(\mathrm{P}-\mathrm{O}-\mathrm{C})\end{array}$ & $\begin{array}{l}0.90-1.23\left(3 \mathrm{t}(\mathrm{m}), 9 \mathrm{H}, H_{3} \mathrm{C} . \mathrm{C}-\mathrm{N} \& 2 \times\right. \\
\left.H_{3} \mathrm{C} . \mathrm{C} . \mathrm{O}\right), 2.64\left(\mathrm{dd}, J_{H H}=7,{ }^{2} J_{P H}=15.8 \mathrm{~Hz},\right. \\
\left.2 \mathrm{H}, H_{2} \mathrm{C}\right), 3.08,3.16\left(\mathrm{~s}, 2 \times 3 \mathrm{H}, 2 \mathrm{NC} H_{3}\right), \\
3.92-4.28\left(3 \mathrm{dq}(\mathrm{m}), 3 \times 2 \mathrm{H}, 2 \times H_{2} \mathrm{CO} \&\right. \\
\left.H_{2} \mathrm{C}-\mathrm{N}\right), 5.17\left(\mathrm{dt}, J_{H H}=7,{ }^{3} J_{P H}=4.4 \mathrm{~Hz},\right. \\
1 \mathrm{H},=\mathrm{CH}) ; \delta_{P}=21.47 \mathrm{ppm} .\end{array}$ & $\begin{array}{l}14.6\left(\mathrm{CH}_{3} \mathrm{C} . \mathrm{N}\right), 16.5\left(\mathrm{CH}_{3} \mathrm{C} .\right. \\
\mathrm{O}), 27.7\left(\mathrm{~d},{ }^{1} \mathrm{~J}_{P C}=111 \mathrm{~Hz},-\right. \\
\left.\mathrm{CH}_{2} \mathrm{P}\right), 28.1,28.6(5-, 7- \\
\left.\left.\mathrm{CH}_{3} \mathrm{~N}\right), 42.2 \mathrm{CH}_{2} \mathrm{~N}\right), 62.3 \\
\left(\mathrm{CH}_{2} \mathrm{O}\right), 64.8(=\mathrm{CH}), 141.7(2- \\
C), 152.6,161.7(4-, 6-C=\mathrm{O}) .\end{array}$ \\
\hline
\end{tabular}

${ }^{a}$ Assigned $\mathrm{N} H$ and $\mathrm{NOH}$ were lost after $\mathrm{D}_{2} \mathrm{O}$ exchange. ${ }^{\mathrm{b}} \mathrm{NMR}$ was run in $\mathrm{d}_{6}$-DMSO; ${ }^{\mathrm{c}} \mathrm{NMR}$ was run in $\mathrm{CDCl}_{3}$. 


\section{Pharmacological evaluation ${ }^{15}$}

The effect on a fifty human tumor cell 1 lines derived from seven-cancer type of some new phosphonates is studied according to the established methods ${ }^{16}$ in terms of structure-activity relationships (SAR). The selection of the tested compounds 5a, 8a, 10a, 17a and 25a was relied on the results of the prediction that carried out, in the earlier stage, by the use of computerassisted molecular modeling (CAMM) for designing the products. ${ }^{16}$ Later on, in vivo the activity of the assigned compounds 5a, 8a, 10a, 17a and 25a were tested against leukemia, melanoma, colon-, renal-, prostate-, ovarian-and breast cancer. Phosphonates 5a, 10a and 17a showed sensitivity against colon cancer (HCT-116 and HCT-15). Also compounds 10a and 17a showed sensitivity against melanoma (SK-MEL-2 and SK-MEL-28). On the other hand, the sensitivity of phosphono substituted 1,2-oxazoles 8a and 25a was noticed against renal cancer (786.0 and UO31) as well as prostate cancer (DU-145) (Table 3).

Table 3. Sensitivity of the Synthesized Compounds 5a, 8a, 10a, 17a and 25a Against Tumor Cell Lines

\begin{tabular}{lccccc}
\hline & \multicolumn{5}{c}{$\log _{10} \mathrm{LC}_{50}$} \\
\cline { 2 - 6 } Panel / cell line & $\mathbf{5 a}$ & $\mathbf{8 a}$ & $\mathbf{1 0 a}$ & $\mathbf{1 7 a}$ & $\mathbf{2 5 a}$ \\
\hline Colon Cancer & & & & & \\
C0L0-205 & ----- & ----- & ---- & ---- & ----- \\
HCT-116 & -4.33 & ----- & -4.44 & -4.44 & ---- \\
HCT-15 & -4.33 & ----- & -4.48 & -4.48 & ----- \\
Melanoma & & & & & \\
M14 & ----- & ----- & ----- & ----- & ----- \\
SK-MEL-2 & ----- & ----- & -4.36 & -4.32 & ---- \\
SK-MEL-28 & ----- & ----- & -4.34 & -4.30 & ---- \\
Renal Cancer & & & & & \\
786.0 & ----- & -4.33 & ----- & ----- & -4.34 \\
RXF-393 & ----- & ----- & ----- & ----- & ----- \\
SN 12C & ----- & ----- & ----- & ---- & ---- \\
UO-31 & ----- & -4.33 & ----- & ---- & -4.36 \\
Prostate Cancer & & & & & \\
DU-145 & ----- & -4.32 & ----- & ----- & -4.39 \\
PC-3 & ----- & ----- & ----- & ----- & ----- \\
\hline
\end{tabular}

Further studies are in progress on the biological activity of this chemical series for the toxicity of these compounds against cell lines and mammals. A comparative screening between the compounds included in Table 3 and the unphosphorylated analogs that obtained from previous communication ${ }^{1}$ to see, whether this activity stems from the heterocylcle or always by the phosphonate rest. Full results of the pharmacological evaluation of these products and other 
related compounds would be published elsewhere.

\section{Conclusion}

Finally, the reactions of $\mathbf{1}$ and $\mathbf{2}$ with the seven WH reagents lead to a methodology for synthesis of condensed and spiro substituted pyrimidine phosphor esters with a biological activity.

\section{Experimental Section}

General Procedures. Melting points are uncorrected. The IR spectra were recorded on a Perkin Elmer 317 Grating IR spectrophotometer, using $\mathrm{KBr}$. The ${ }^{1} \mathrm{H}$ and ${ }^{13} \mathrm{C} \mathrm{NMR}$ spectra were measured on a Joel E.C.A-500 MHz instrument using $\mathrm{SiMe}_{4}$ as an internal reference. The ${ }^{31} \mathrm{P}$ NMR spectra were recorded with the same instrument, relative to external $\mathrm{H}_{3} \mathrm{PO}_{4}(85 \%)$. The mass spectra were performed on a Joel JMS-A X 500 spectrometer. Elemental analyses were carried out at the Microanalysis Laboratory, Cairo University, Cairo, Egypt. The appropriate precautions in handling moisture-sensitive compounds were observed. Solvents were dried by standard techniques. The substrates violuric acid (1) ${ }^{13}$ and 1,3-dimethyl alloxan-5-oxime (2) ${ }^{14}$ were prepared according to the reported method.

\section{Reactions of alloxan-5-oximes 1 and 2 with WH Reagents 3a,b; 9; 11a,b; 14 and 22 General procedure}

To a stirred solution of $8.6 \mathrm{mmol}$ of the appropriate phosphonate $\mathbf{3 a} \mathbf{a}, \mathbf{b} ; \mathbf{9} ; \mathbf{1 1} \mathbf{a}, \mathbf{b} ; \mathbf{1 4}$ or 22 and 25 mmol of $\mathrm{Na}$ in $15 \mathrm{~mL}$ dry EtOH was added slowly to a solution of $7.64 \mathrm{mmol}$ of oxime 1 (or 2) in $20 \mathrm{~mL}$ EtOH at $0{ }^{\circ} \mathrm{C}$. The resulting mixture was stirred at the reflux temperature up to the consumption of the starting oxime $((\approx 24 \mathrm{~h}$, TLC). After concentration of the solvent, $20 \mathrm{~mL}$ of distilled water was added and the solution was acidified with $\mathrm{HCl}(1 \mathrm{~N})$ until the $\mathrm{pH}$ of the reaction mixture became acidic. The resulting solution was extracted with $(3 \mathrm{x} 50 \mathrm{~mL})$ isopropyl alcohol (or AcOEt). The combined organic phase was dried over anhydrous $\mathrm{MgSO}_{4}$ and the solvent was removed under reduced pressure. The resulting residue was chromatographed on silica gel by using $n$-hexane / AcOEt as eluents to give the products 5a,b, 8a,b, 10a,b, 13a,b, 17a,b, 21a,b, 25a,b and 27a,b. Percentage yields; physical and spectral data of the products are listed in Tables 1 and 2 . 


\section{References}

1. Abdou, W. M.; Barghash, R. F. Trends in Heterocycles 2005, 10, 57.

2. (a) Ghosh, R.; Singh, B. R. Indian J. Chem. 1982, 21A, 20. (b) Ghosh, R.; Singh, B. R. Indian J. Chem. 1982, 21A, 23. (c) Ghosh, R.; Singh, B. R. Indian J. Chem. 1980, 19A, 1102.

3. Ghatak, N.; Dutt, S. J. Indian Chem. Soc. 1928, 5, 665.

4. Maryanoff, B. E.; Reitz, A. B. Chem. Rev. 1989, 89, 383.

5. (a) Rieker, A.; Rundel, W.; Kessler, H. Z. Naturforsch. 1969, 24, 547. (b) Abdou, W. M.; ElKhoshnieh, Y. O. Synth. Comm. 1999, 29, 2664.

6. Pudovik, A. N.; Lebedera, N. M. Doklady Akad. Nauk SSSR. 1953, 90, 799; Chem. Abstr. 1956, 50, 2429. Patia, S.; Schwartz, A. J. Org. Chem. 1960, 25, 1232.

7. (a) Mishriky, N.; Asaad, F. M.; Ibrahim, Y. A.; Girgis, A. S. J. Chem. Res. (S) 1997, 438; (M), 2758. (b) El-Kateb, A. A.; Abdel-Malek, H. A. Phosphorus, Sulfur and Silicon, 1996, 112, 41. (c) Abdou, W. M.; Salem, M. A. I.; Sediek, A. A. Montsch. Chem. 2003, 134, 1373.

8. (a) Boulos, L. S.; Arsanious, M. H. N. Phosphorus, Sulfur, and Silicon 1994, 89, 185. (b) Boulos, L. S.; Arsanious, M. H. N.; Eldin, N. K. Phosphorus, Sulfur, and Silicon 1997, 122, 49. (c) Mercey, M.; Toube, P. J. Chem. Res. Synop. 1987, 13. (d) Yakout, E. M. A.; Giurgius, D. B.; Boulos, L. S. Phosphorus, Sulfur, and Silicon 1999, 148, 177.

9. Coppola, G. M.; Hartmann, G. E.; Pister, O. R. J. Org. Chem. 1976, 41, 825.

10. Latham, E. J.; Murphy, S. M.; Stanforth, S. P. Tetrahedron, 1995, 51, 10385.

11. (a) Schweizer, E. E.; Copay, C. M. J. Org. Chem. 1972, 37, 1561. (b) Yavari, I., Djahaniani, H., Maghsoodlau, M. T. Hazeri, N. J. Chem. Research (S), 1999, 382.

12. (a) Zbiral, E. Tetrahedron Lett. 1970, 5107. (b) Krespan, C. G. J. Am. Soc. 1961, 83, 3432.

13. Ceresole, M. Chem. Ber. 1883, 16, 1134.

14. Leermakers, P. A. J. Am. Chem. Soc. 1958, 80, 5663.

15. The anti-tumor screening was carried out at the Central Lab of Biology, Banha University, Banha, Egypt.

16. Boyd, M. R. Principles and Practices of Oncology, 1989, 3, 1. (b) Alley, M. C.; Scudiero, D. C.; Monks, A.; Flugsey, M. L.; Czerwinski, M. J.; Fine, D. L.; Abbott, B. J.; Mayo, J. C.; Shoemaker, R. H.; Boyd, M. R. Cancer Res. 1988, 48, 589.

17. Poroikov, V. V.; Filimonov, D. A; Ihlenfeld, W. D.; Gloriozova, T. A.; Lagunin, A. A.; Borodina, Yu. V.; Stepanchikova, A. V.; Nicklaus, M. C. PASS Biological Activity Spectrum Predictions in the Enhanced Open NCI Database Browser J. Chem. Inf. Comput. Sci. 2003, 43, 228; Visit: Website: http://www.ibmc.msk.ru/PASS. 